\title{
Emotional modulation of synapses, circuits and memory
}

\author{
Jonathan E. Ploski and Christa K. Mclntyre* \\ Cognition and Neuroscience Program, School of Behavioral and Brain Sciences, The University of Texas at Dallas, Richardson, TX, USA \\ *Correspondence: christa.mcintyre@utdallas.edu \\ Edited and reviewed by: \\ Nuno Sousa, University of Minho, Portugal
}

Keywords: amygdala, fear conditioning, extinction, reconsolidation, oscillations, synapse allocation

Emotion is a powerful tool for change in the central nervous system. Whereas, most long-term memories are stored after practice or rehearsal, an emotionally arousing memory can be consolidated after a single experience. Research examining the influence of emotion on synaptic function provides a window of opportunity for exploring the mechanisms of memory consolidation during the minutes to hours after a single, well-remembered experience. It can also shed light on numerous psychiatric conditions, bringing the field closer to preventing or treating those conditions that stem from traumatic memories. This research topic brings together leading experts who share their recent findings and perspectives on how emotion may influence brain function.

In this issue, four articles review or describe evidence of a specialized influence of emotion on synaptic function. Sheena Josselyn's laboratory previously found that neurons with high levels of cAMP Responsive Element Binding Protein (CREB) at the time of training are preferentially allocated to the memory trace (Han et al., 2007). In this issue, Sargin et al. (2013) provide a cellular mechanism as to why this may be. They report that overexpression of CREB within neurons of the lateral nucleus of the amygdala (LA), leads to an increase in dendritic spine density, whereas LA neurons with low CREB activity exhibit a decrease in dendritic spine density. These data support the hypothesis that CREB may increase a neuron's propensity for being included in a memory trace by increasing dendritic spine density.

Young and Williams addressed the issue of lateralization of amygdala recruitment during memory consolidation. Imaging studies in humans indicate that activity in the right amygdala is associated with aversive memories (Morris et al., 1999). Others indicate that the left amygdala is preferentially involved in encoding memories that have a positive valence (Zalla et al., 2000; Hamann et al., 2002). In this issue, Young and Williams (2013) examine expression of a marker of synaptic plasticity in the rat amygdala. They report that the synaptic plasticity-related protein Arc is specifically elevated in the right amygdala following training on an aversive task, and in the left amygdala following training on an appetitive task. Considering that Arc expression in the amygdala is necessary for consolidation of conditioned fear (Ploski et al., 2008), these findings indicate that, memoryrelated synaptic plasticity in the amygdala is lateralized and valence-dependent.

Headley and Paré (2013) add a temporal dimension to this research topic. They review literature on temporal synchrony of firing across brain regions involved in emotional memory.
They describe gamma oscillations and emotional memory, citing recent reports of enhanced gamma oscillations in the neocortex and amygdala during emotional situations, and evidence that gamma oscillations have predictive value for synaptic plasticity and emotional memory.

Grønli et al. (2013) take a different view on the effects of emotion on memory and plasticity. These authors review the cellular and molecular evidence that stress may impair cognition by interfering with sleep. They describe the importance of sleep for the cellular and molecular processes that contribute to memory consolidation, and suggest that stress that interferes with sleep is associated with deficient synaptic plasticity and impaired cognitive performance.

Several of the contributions to this issue examined synaptic changes related to extinction of conditioned fear in rats. Both post-traumatic stress disorder (PTSD) and obsessive compulsive disorder (OCD) are characterized by avoidance of stimuli that are perceived as threatening, even in the absence of real danger. Impaired extinction of conditioned fear could contribute to the persistence of maladaptive behaviors seen in these disorders (Milad et al., 2008, 2013). It was recently demonstrated that vagus nerve stimulation (VNS) enhances extinction of conditioned fear in rats (Peña et al., 2013). In this issue, (Peña et al., 2014) report that extinction-enhancing VNS reverses synaptic depression in the infralimbic prefrontal cortex basolateral amygdala pathway, a circuit that is implicated in extinction memory (Sierra-Mercado et al., 2011). Collectively, these findings suggest that VNS could be paired with exposure therapy to facilitate extinction of conditioned fear and reverse pathological synaptic function seen in anxiety disorders. Another contribution investigated the effects of deep brain stimulation (DBS) on the extinction circuitry. In 2010, several groups reported that stimulation of the ventral capsule/ventral striatum reduced symptoms of OCD in refractory patients (Denys et al., 2010; Goodman et al., 2010; Greenberg et al., 2010). In rats, stimulation of the ventral striatum enhances extinction of conditioned fear (RodriguezRomaguera et al., 2012). In this issue, research from Greg Quirk's lab demonstrates that extinction-enhancing stimulation of the ventral striatum increases expression of the brain-derived neurotrophic factor protein (BDNF) in the medial prefrontal cortex, indicating that the clinical benefits of DBS may be mediated by BDNF-associated synaptic changes in the extinction pathway (Do-Monte et al., 2013).

One of the limitations of exposure therapy is that conditioned fear can return even after successful extinction learning. In 
2009, Monfils and colleagues reported evidence that pairing of a brief retrieval trial with extinction training produced a persistent reduction of conditioned fear that was not susceptible to spontaneous recovery, reinstatement, or renewal of fear (Monfils et al., 2009). This effect is now referred to as "reconsolidation updating" because the brief retrieval trial is thought to destabilize the memory trace. The destabilized conditioned fear memory trace is thought to be modified by the extinction training. However, because some laboratories do not observe persistent reductions in fear using the reconsolidation-extinction approach, researchers in the laboratories of Marie Monfils and Hongjo Lee collaborated to determine whether orienting phenotype affects the persistence of the fear reduction. These authors contributed two articles to this issue. Both of these studies distinguished rats that direct attention to a conditioned stimulus during appetitive conditioning (orienters) from rats that do not (non-orienters). Expression of conditioned fear $24 \mathrm{~h}$ after training is greater in non-orienters. However, pairing a brief retrieval trial with an extinction session prevents spontaneous recovery of fear in both phenotypes, indicating that orienting phenotype is not a boundary condition that would interfere with the permanence of the effect (Olshavsky et al., 2013a). In contrast, orienting phenotype has a significant effect on the persistence of reconsolidation updating when the conditioned stimulus is appetitive. Conditioned responding spontaneously recovers only in the non-orienting phenotype (Olshavsky et al., 2013b).

Historically, reconsolidation updating and extinction learning have been considered separate processes. However, with the discovery and development of the reconsolidation-extinction paradigm (Monfils et al., 2009), it has become apparent that there is overlap between reconsolidation and extinction processes. In this issue Flavell and colleagues review what is known about the cellular and molecular mechanisms that govern the reactivationdependent destabilization of memory and how these processes influence the permanent weakening of memory. Considering not all memories become destabilized upon retrieval, and therefore are resistant to being modified, this area of research holds great promise for the development of treatments targeting pathological memory (Flavell et al., 2013).

Roesler and colleagues sought to enhance extinction of conditioned fear in rats but instead found that intra-dorsal hippocampus infusions of the phosphodiesterase type 4 (PDE4) inhibitor, rolipram, during extinction training, can switch the behavioral outcome from extinction to enhanced fear (Roesler et al., 2014). These intriguing findings indicate that inhibition of PDE4 during extinction training may promote reconsolidation update mechanisms and/or inhibit extinction learning.

Extensive evidence supports the role of de novo protein synthesis in the consolidation and reconsolidation of memory. In addition, there is accumulating evidence indicating that coordinated protein degradation via the ubiquitin-proteasome system (UPS) is required for these processes. For example, inhibition of the UPS disrupts the consolidation of memory and the destabilization phase of memory updating (Jarome and Helmstetter, 2013). Recently Kwapis et al. (2014) demonstrated that protein degradation via the UPS, within the LA was critical for the consolidation of delay fear conditioning. In this issue, Reis et al.
(2013) extend these findings by demonstrating that pharmacologically inhibiting the UPS, with the proteasome inhibitor clasto-lactacystin- $\beta$-lactone $(\beta$-lac) within the prefrontal cortex, selectively disrupts trace fear conditioning, while leaving delay fear conditioning intact. These findings further support the role of the PFC in trace, but not delay conditioning and underscore the wide spread importance of UPS mediated protein degradation in learning and memory phenomena.

Animal models of trauma and anxiety disorders are helpful in translating these discoveries to therapies. Berardi et al. (2014) present a rodent model of PTSD that meets cognitive and emotional criteria for diagnosis according to the most recent version of the Diagnostic and Statistical Manual of Mental Disorders (DSM-5). They found that rats exposed to inescapable footshocks and housed in isolation exhibited fear of the context that was associated with the shock for 56 days. These rats also exhibited long-lasting alterations in social interactions and exploration on the elevated plus maze. Such advancements in animal models of PTSD may provide new avenues for exploring the effects of traumatic experiences on the brain as well as opportunities for testing possible therapies.

\section{ACKNOWLEDGMENT}

The editors thank the authors who contributed their new ideas and research findings to this research topic.

\section{REFERENCES}

Berardi, A., Trezza, V., Palmery, M., Trabace, L., Cuomo, V., and Campolongo, P. (2014). An updated animal model capturing both the cognitive and emotional features of post-traumatic stress disorder (PTSD). Front. Behav. Neurosci. 8:142. doi: 10.3389/fnbeh.2014.00142

Denys, D., Mantione, M., Figee, M., van den Munckhof, P., Koerselman, F., Westenberg, H., et al. (2010). Deep brain stimulation of the nucleus accumbens for treatment-refractory obsessive-compulsive disorder. Arch. Gen. Psychiatry 67, 1061-1068. doi: 10.1001/archgenpsychiatry.2010.122

Do-Monte, F. H., Rodriguez-Romaguera, J., Rosas-Vidal, L. E., and Quirk, G. J. (2013). Deep brain stimulation of the ventral striatum increases BDNF in the fear extinction circuit. Front. Behav. Neurosci. 7:102. doi: 10.3389/fnbeh.2013.00102

Flavell, C. R., Lambert, E. A., Winters, B. D., and Bredy, T. W. (2013). Mechanisms governing the reactivation-dependent destabilization of memories and their role in extinction. Front. Behav. Neurosci. 7:214. doi: 10.3389/fnbeh.2013.00214

Goodman, W. K., Foote, K. D., Greenberg, B. D., Ricciuti, N., Bauer, R., Ward, H., et al. (2010). Deep brain stimulation for intractable obsessive compulsive disorder: pilot study using a blinded, staggered-onset design. Biol. Psychiatry 67, 535-542. doi: 10.1016/j.biopsych.2009.11.028

Greenberg, B. D., Rauch, S. L., and Haber, S. N. (2010). Invasive circuitry-based neurotherapeutics: stereotactic ablation and deep brain stimulation for OCD. Neuropsychopharmacology 35, 317-336. doi: 10.1038/npp.2009.128

Grønli, J., Soulé, J., and Bramham, C. R. (2013). Sleep and protein synthesisdependent synaptic plasticity: impacts of sleep loss and stress. Front. Behav. Neurosci. 7:224. doi: 10.3389/fnbeh.2013.00224

Hamann, S. B., Ely, T. D., Hoffman, J. M., and Kilts, C. D. (2002). Ecstasy and agony: activation of the human amygdala in positive and negative emotion. Psychol. Sci. 13, 135-141. doi: 10.1111/1467-9280.00425

Han, J. H., Kushner, S. A., Yiu, A. P., Cole, C. J., Matynia, A., Brown, R. A., et al. (2007). Neuronal competition and selection during memory formation. Science 316, 457-460. doi: 10.1126/science.1139438

Headley, D. B., and Paré, D. (2013). In sync: gamma oscillations and emotional memory. Front. Behav. Neurosci. 7:170. doi: 10.3389/fnbeh.2013.00170

Jarome, T. J., and Helmstetter, F. J. (2013). The ubiquitin-proteasome system as a critical regulator of synaptic plasticity and long-term memory formation. Neurobiol. Learn. Mem. 105, 107-116. doi: 10.1016/j.nlm.2013.03.009 
Kwapis, J. L., Jarome, T. J., Lee, J. L., Gilmartin, M. R., and Helmstetter, F. J. (2014). Extinguishing trace fear engages the retrosplenial cortex rather than the amygdala. Neurobiol. Learn. Mem. 113, 41-54. doi: 10.1016/j.nlm.2013.09.007

Milad, M. R., Furtak, S. C., Greenberg, J. L., Keshaviah, A., Im, J. J., Falkenstein, M. J., et al. (2013). Deficits in conditioned fear extinction in obsessive-compulsive disorder and neurobiological changes in the fear circuit. JAMA Psychiatry 70, 608-618, quiz 554. doi: 10.1001/jamapsychiatry.2013.914

Milad, M. R., Orr, S. P., Lasko, N. B., Chang, Y., Rauch, S. L., and Pitman, R. K. (2008). Presence and acquired origin of reduced recall for fear extinction in PTSD: results of a twin study. J. Psychiatr. Res. 42, 515-520. doi: 10.1016/j.jpsychires.2008.01.017

Monfils, M. H., Cowansage, K. K., Klann, E., and LeDoux, J. E. (2009). Extinctionreconsolidation boundaries: key to persistent attenuation of fear memories. Science 324, 951-955. doi: 10.1126/science.1167975

Morris, J. S., Ohman, A., and Dolan, R. J. (1999). A subcortical pathway to the right amygdala mediating "unseen" fear. Proc. Natl. Acad. Sci. U.S.A. 96, 1680-1685. doi: 10.1073/pnas.96.4.1680

Olshavsky, M. E., Jones, C. E., Lee, H. J., and Monfils, M. H. (2013a). Appetitive behavioral traits and stimulus intensity influence maintenance of conditioned fear. Front. Behav. Neurosci. 7:179. doi: 10.3389/fnbeh.2013.00179

Olshavsky, M. E., Song, B. J., Powell, D. J., Jones, C. E., Monfils, M. H., and Lee, H. J. (2013b). Updating appetitive memory during reconsolidation window: critical role of cue-directed behavior and amygdala central nucleus. Front. Behav. Neurosci. 7:186. doi: 10.3389/fnbeh.2013.00186

Peña, D. F., Childs, J. E., Willett, S., Vital, A., McIntyre, C. K., and Kroener, S. (2014). Vagus nerve stimulation enhances extinction of conditioned fear and modulates plasticity in the pathway from the ventromedial prefrontal cortex to the amygdala. Front. Behav. Neurosci. 8:327. doi: 10.3389/fnbeh.2014.00327

Peña, D. F., Engineer, N. D., and McIntyre, C. K. (2013). Rapid remission of conditioned fear expression with extinction paired with vagus nerve stimulation. Biol. Psychiatry 73, 1071-1077. doi: 10.1016/j.biopsych.2012.10.021

Ploski, J. E., Pierre, V. J., Smucny, J., Park, K., Monsey, M. S., Overeem, K. A., et al. (2008). The activity-regulated cytoskeletal-associated protein (Arc/Arg3.1) is required for memory consolidation of pavlovian fear conditioning in the lateral amygdala. J. Neurosci. 28, 12383-12395. doi: 10.1523/JNEUROSCI.166208.2008

Reis, D. S., Jarome, T. J., and Helmstetter, F. J. (2013). Memory formation for trace fear conditioning requires ubiquitin-proteasome mediated protein degradation in the prefrontal cortex. Front. Behav. Neurosci. 7:150. doi: $10.3389 /$ fnbeh. 2013.00150
Rodriguez-Romaguera, J., Do Monte, F. H., and Quirk, G. J. (2012). Deep brain stimulation of the ventral striatum enhances extinction of conditioned fear. Proc. Natl. Acad. Sci. U.S.A. 109, 8764-8769. doi: 10.1073/pnas. 1200782109

Roesler, R., Reolon, G. K., Maurmann, N., Schwartsmann, G., Schröder, N., Amaral, O. B., et al. (2014). A phosphodiesterase 4-controlled switch between memory extinction and strengthening in the hippocampus. Front. Behav. Neurosci. 8:91. doi: 10.3389/fnbeh.2014.00091

Sargin, D., Mercaldo, V., Yiu, A. P., Higgs, G., Han, J. H., Frankland, P. W., et al. (2013). CREB regulates spine density of lateral amygdala neurons: implications for memory allocation. Front. Behav. Neurosci. 7:209. doi: 10.3389/fnbeh.2013.00209

Sierra-Mercado, D., Padilla-Coreano, N., and Quirk, G. J. (2011). Dissociable roles of prelimbic and infralimbic cortices, ventral hippocampus, and basolateral amygdala in the expression and extinction of conditioned fear. Neuropsychopharmacology 36, 529-538. doi: 10.1038/npp. 2010.184

Young, E. J., and Williams, C. L. (2013). Differential activation of amygdala Arc expression by positive and negatively valenced emotional learning conditions. Front. Behav. Neurosci. 7:191. doi: 10.3389/fnbeh.2013.00191

Zalla, T., Koechlin, E., Pietrini, P., Basso, G., Aquino, P., Sirigu, A., et al. (2000). Differential amygdala responses to winning and losing: a functional magnetic resonance imaging study in humans. Eur. J. Neurosci. 12, 1764-1770. doi: 10.1046/j.1460-9568.2000.00064.x

Conflict of Interest Statement: The authors declare that the research was conducted in the absence of any commercial or financial relationships that could be construed as a potential conflict of interest.

Received: 15 January 2015; accepted: 01 February 2015; published online: 19 February 2015.

Citation: Ploski JE and McIntyre CK (2015) Emotional modulation of synapses, circuits and memory. Front. Behav. Neurosci. 9:35. doi: 10.3389/fnbeh.2015.00035 This article was submitted to the journal Frontiers in Behavioral Neuroscience. Copyright (C) 2015 Ploski and McIntyre. This is an open-access article distributed under the terms of the Creative Commons Attribution License (CC BY). The use, distribution or reproduction in other forums is permitted, provided the original author(s) or licensor are credited and that the original publication in this journal is cited, in accordance with accepted academic practice. No use, distribution or reproduction is permitted which does not comply with these terms. 